\title{
Evaluating Guanfacine Hydrochloride in the Treatment of Attention Deficit Hyperactivity Disorder (ADHD) in Adult Patients: Design, Development and Place in Therapy
}

\author{
Toyosaku Ota (iD) ${ }^{1,2}$ \\ Kazuhiko Yamamuro' \\ Kosuke Okazaki ${ }^{\prime}$ \\ Toshifumi Kishimoto' \\ 'Department of Psychiatry, Nara Medical \\ University, Kashihara, Japan; ${ }^{2}$ Faculty of \\ Nursing, School of Medicine, Nara \\ Medical University, Kashihara, Japan
}

\begin{abstract}
Attention-deficit/hyperactivity disorder (ADHD) is characterized by ageinappropriate and impairing levels of inattention, hyperactivity, or impulsivity, or a combination of these characteristics. It is estimated to affect around $4 \%$ of adults worldwide. In the past few decades, prescriptions for ADHD drugs (psychostimulants and nonpsychostimulants) have increased significantly. However, the efficacy and safety of adult ADHD medications remains controversial. Guanfacine extended-release (GXR) is a nonpsychostimulant ADHD drug that is a selective $\alpha 2 \mathrm{~A}$-adrenergic receptor agonist, first approved for treatment of adult ADHD in Japan in June 2019. Our aim was to provide an overview of GXR pharmacology and review the studies on efficacy and safety that have been conducted in adults with ADHD. The beneficial actions of guanfacine are thought to be attributed to the strengthening of prefrontal cortical network connections, which regulate attention, emotion, and behavior via the activity at post-synaptic $\alpha 2 \mathrm{~A}$ receptors. Current evidence of GXR efficacy and safety suggests that GXR is an effective monotherapy treatment option for adults with ADHD.
\end{abstract}

Keywords: attention-deficit/hyperactivity disorder, pharmacotherapy, guanfacine, adults, nonstimulant

\section{Introduction}

Attention-deficit/hyperactivity disorder (ADHD) is characterized by ageinappropriate and impairing levels of inattention, hyperactivity, or impulsivity, or a combination of these characteristics. ${ }^{1}$ It is estimated to affect $7-10 \%$ of children ${ }^{2}$ and about $4 \%$ of adults. ${ }^{3}$ Available pharmacological treatments for ADHD include psychostimulant (eg methylphenidate and amphetamine) and non-psychostimulant medications (eg atomoxetine and $\alpha 2$-agonists). In the past few decades, prescriptions for ADHD drugs have significantly increased globally. ${ }^{4,5}$ Despite clinical guidelines recommending the use of these drugs for treating $\mathrm{ADHD},{ }^{6-9}$ the treatment guidelines for adult ADHD are developing.

Guanfacine extended-release (GXR) is a nonstimulant, selective, $\alpha 2 \mathrm{~A}$ adrenergic receptor agonist. The guanfacine extended-release formulation has been available in the United States since 2009. The GXR is approved by the US Food and Drug Administration for the treatment of ADHD in patients aged 6-17
Correspondence: Toyosaku Ota Department of Psychiatry, Nara Medical University, 840 Shijyo-Cho, Kashihara

Nara, 634-8522, Japan

Tel $+8|-742-22-305|$

Fax $+81-742-22-3854$

Email toyosaku@naramed-u.ac.jp 
years, either as monotherapy or an adjunctive therapy for stimulants. However, there have not been sufficient examinations of efficacy and safety of this drug in adults with ADHD. In fact, GXR for adults was not included in the comprehensive 2018 systematic review and meta-analysis of medications for ADHD; ${ }^{10}$ moreover, it has not been included in the current international guidelines. ${ }^{11}$

In Japan, GXR was first approved for treatment of adults with ADHD in June 2019. Therefore, we sought to provide an overview of GXR pharmacology and review studies that have evaluated the efficacy and safety of GXR for the treatment of adults with ADHD.

\section{Pharmacology of GXR}

Guanfacine differs from clonidine in that it is a phenylacetylguanidine derivative $\mathrm{e}^{12}$ and is more selective in its $\alpha 2$ agonism. ${ }^{13}$ Like the action of clonidine, it lowers blood pressure by activating brainstem receptors, which leads to decreased sympathetic tone. ${ }^{14}$ Guanfacine's beneficial effects on ADHD are thought to occur via its agonism at $\alpha 2 \mathrm{~A}$-adrenoceptors in the prefrontal cortex. The prefrontal cortex is the primary brain area implicated in ADHD, and the prefrontal dysfunction may underlie symptoms of inattentiveness, impulsivity, and excessive motor activity. ${ }^{15}$ Furthermore, the prefrontal cortex is important for executive functions (eg planning, decision making, judgment, and response inhibition).

Both norepinephrine and dopamine are crucial for prefrontal cortex functions. ${ }^{16}$ Sufficient levels of norepinephrine are required for optimal working memory and attention; however, excessive norepinephrine release (eg during stress) can also impair cognitive functions. ${ }^{15}$ Optimal levels of norepinephrine activate $\alpha 2 \mathrm{~A}-$ adrenoceptors, whereas excessive levels lead to stimulation of lower affinity $\alpha 1$-adrenoceptors. Experimentally, blocking prefrontal $\alpha 2 \mathrm{~A}$-adrenoreceptors induces motor hyperactivity in primates. Guanfacine has been shown to enhance attention and hyperactivity in animal models of ADHD. ${ }^{17}$ Guanfacine has also been shown to extend the activity of the frontal cortex in both rats ${ }^{18}$ and humans. ${ }^{19}$

The GXR tablet is formulated as a single daily extended-release matrix tablet, ${ }^{20}$ that should be swallowed whole, instead of chewed, crushed, or split, to increase the rate of guanfacine release. Guanfacine is absorbed effectively from GXR tablets that are taken orally; ${ }^{21}$ the time to peak plasma concentration is approximately 5 hours in children and adolescents with ADHD. ${ }^{21}$ However, consumption of a food has an effect on exposure to guanfacine via GXR tablets, with a $75 \%$ increase in peak concentration of plasma and $40 \%$ increase in area under the concentration-time curve of plasma when exposed to a high-fat breakfast. ${ }^{21}$ Therefore, exposure to a high-fat food should be shunned.

In plasma, guanfacine is approximately $70 \%$ bound to plasma proteins. ${ }^{21}$ Guanfacine is mainly metabolized by hepatic cytochrome p450 (CYP) 3A4 microsomal enzymes, and exposure to guanfacine has the potential to be increased by the simultaneous use of CYP $3 \mathrm{~A} 4$ inhibitors (eg itraconazole, clarithromycin, fluvoxamine, and grapefruit juice) or decreased by the simultaneous use of CYP $3 \mathrm{~A} 4$ inducers (eg rifampin, carbamazepine, phenytoin, and St. John's Wort). ${ }^{21}$ However, CYP enzymes are not inhibited or induced by guanfacine. ${ }^{21}$ The elimination half-life of guanfacine following GXR administration is $16-17$ hours, ${ }^{20}$ which enables a single daily administration of GXR. Furthermore, $50 \%$ of one GXR dose is excreted unaltered within the urine. ${ }^{20}$ In addition, it is suggested that guanfacine may affect the pharmacokinetics of valproic acid and lead to a significant increase in valproate plasma levels when used concurrently with this agent.

Guanfacine reduces sympathetic nerve impulses, which leads to reduced sympathetic outflow and follow-on decrease in vasomotor tone and heart rate. Additionally, guanfacine selectively binds to postsynaptic $\alpha 2 \mathrm{~A}$ adrenoreceptors in the prefrontal cortex and has been theorized to enhance delay-related neural firing at the prefrontal cortex. This affects behavioral inhibition and working memory, which improves symptoms associated with ADHD. Similar to norepinephrine, guanfacine acts on $\alpha 2 \mathrm{~A}$-receptors within the cortex to reinforce the signalto-noise ratio of surrounding stimuli, which is assumed to enhance the power to focus on a specific stimulus "above the noise" during times of low arousal. ${ }^{22}$

\section{GXR for Treating Adult ADHD}

Butterfield et al studied 26 adults with a diagnosis of ADHD who were 19-62 years of age. ${ }^{23}$ The study's primary outcome measures were the ADHD Rating Scale (ADHD-RS) and the Clinical Global Impression-Severity (Table 1). Patients were randomly assigned to two treatment groups, where their existing psychostimulant treatment regimen was supplemented with either a titrated dose (1-6 mg) of GXR or placebo over a 10-week trial period. The data were analyzed using a standard mixed-model analysis of variance procedure, and patients in both groups 
Table I Characteristics of GXR Study for Adult ADHD

\begin{tabular}{|l|l|l|l|l|l|l|}
\hline Author, Year & Duration & $\begin{array}{l}\text { Diagnosis of } \\
\text { ADHD }\end{array}$ & $\begin{array}{l}\text { Mean Age } \\
\text { (SD) }\end{array}$ & $\begin{array}{l}\text { Male } \\
\%\end{array}$ & $\begin{array}{l}\text { Intervention (No. } \\
\text { Dose) }\end{array}$ & $\begin{array}{l}\text { Primary Outcome } \\
\text { Measures }\end{array}$ \\
\hline $\begin{array}{l}\text { Butterfield, } \\
2016\end{array}$ & 10 weeks & DSM-IV-TR & $37.5(12.2)$ & $46.2 \%$ & $\begin{array}{l}\text { Placebo } \\
\text { GXR I-6mg } \\
\text { Total: 26 }\end{array}$ & ADHD-RS, CGI-S \\
\hline Iwanami, 2020a & 12 weeks & DSM-5 & $31.1(8.1)$ & $66.0 \%$ & $\begin{array}{l}\text { Placebo (100) } \\
\text { GXR (10I) 4-6mg }\end{array}$ & ADHD-RS \\
\hline Iwanami, 2020b & 50 weeks & DSM-5 & $33.1(9.4)$ & $68.1 \%$ & GXR (19I) 4-6mg & ADHD-RS \\
\hline
\end{tabular}

showed significant improvement in symptoms and functioning during the trial. There was no difference between the treatments in terms of their efficacy, safety, or tolerability.

Iwanami et al assessed GXR efficacy and safety in adults with ADHD in a Phase 3 , double-blind, placebocontrolled study that included Japanese patients with ADHD aged $\geq 18$ years. $^{24}$ Patients were administered GXR $(\mathrm{n}=101)$ or placebo $(\mathrm{n}=100)$, titrated from $2 \mathrm{mg} /$ day to 4-6 mg/day with dose optimization during 5 weeks, followed by $4-6 \mathrm{mg} /$ day in maintaining the dose during 5 weeks, then tapered to $2 \mathrm{mg} /$ day for 2 weeks (Table 1 ). Compared with placebo, GXR treatment showed a significant decrease in ADHD-RS total score. Additionally, treatment with GXR showed significantly greater improvements in two subscale (inattention and hyperactivity-impulsivity) scores of the ADHD-RS, Clinical Global Impression-Improvement scale scores, and Patient Global Impression-Improvement scale scores. However, there were more participants in the GXR group compared with participants in the placebo group who reported treatment-emergent adverse events and who discontinued GXR because of treatment-emergent adverse events. The main adverse events in the GXR group were somnolentia, mouth dryness, reduced blood pressure, nasopharyngitis, dizziness upon standing, and astriction, although most treatment-emergent adverse events were mild to moderate in regard to severity. Therefore, the study concluded that GXR monotherapy improves ADHD symptoms in Japanese adults with ADHD without major safety concerns.

In another study, the safety and efficacy of prolonged pharmaceutical therapy of GXR in adults with ADHD were assessed by Iwanami et al. ${ }^{25}$ In this open-label, prolonged period, phase 3, extension study conducted in Japan, 150 participants who had continued from a double-blind period, and 41 newly attended participants were administered single daily GXR from $2 \mathrm{mg}$ /day during 50 weeks (maintenance dose 4-6 mg/day) (Table 1). One hundred and eighty of 191 participants reported more than one treatment-emergent adverse event and 38 of 191 participants discontinued because of treatment-emergent adverse events. Most treatment-emergent adverse events were mild to moderate with regard to severity; two were considered serious treatmentemergent adverse events but there were no deaths. Treatment-emergent adverse events that were reported by more than $10 \%$ of participants were somnolentia, mouth dryness, nasopharyngitis, decreased blood pressure, dizziness upon standing, cardiac slowing, lassitude, astriction, and dizziness. The ADHD-RS scores (total, inattention subscale, and hyperactivity-impulsivity subscale), Adult ADHD Quality of Life Questionnaire total scores, and executive functioning showed significant improvement in participants in double-blind trial and newly enrolled participants at the final point, and there was also an increase in the proportion of patients who showed considerable improvement in Clinical Global Impression-Improvement scale scores and Patient Global Impression-Improvement scale scores. Therefore, results demonstrated that there were no major safety issues for prolonged pharmaceutical therapy of GXR for adults with ADHD, and patients showed significant ameliorations in ADHD symptoms, quality of life, and executive function following long-term GXR monotherapy.

Taking it into consideration, rebound hypertension is a potential concern with sudden discontinuation of alpha-2 agonists. Persistent blood pressure increases of up to 10 $\mathrm{mmHg}$ have been observed in a few individuals at 30 days post-discontinuation. ${ }^{26}$ All reviewed trials had a dose tapering period following the maintenance phase, in keeping with the manufacturer's recommendations for gradual dosage decrements of no more than $1 \mathrm{mg}$ every 3-7 days when tapering off GXR. $^{26}$ 


\section{GXR for Treating Comorbid ASD and Tic Disorder}

Scahill et al assessed GXR efficacy and safety in autism spectrum disorder with hyperactivity. ${ }^{27}$ This study was a five-site randomized, double-blind placebo-controlled, fixed-flexible dose clinical trial conducted by the Research Units on Pediatric Psychopharmacology Autism Network. Sixty-two subjects were randomly assigned to GXR $(n=30)$ or placebo $(n=32)$ for 8 weeks. The GXR group showed a $43.6 \%$ decline in scores on the Aberrant Behavior Checklist-hyperactivity subscale compared with a $13.2 \%$ decrease in the placebo group. The most common adverse events included drowsiness, fatigue, and decreased appetite. There were no significant changes on ECG in either group. For subjects in the GXR group, blood pressure declined in the first 4 weeks, with return nearly to baseline by endpoint (week 8). Pulse rate showed a similar pattern but remained lower than baseline at endpoint. Therefore, the study concluded that GXR appears to be safe and effective for reducing hyperactivity, impulsiveness, and distractibility in children with autism spectrum disorder although there was no effects for autism spectrum symptoms.

In the meta-analysis of treatment for children with ADHD with comorbid tic disorder, methylphenidate, alpha-2 agonists, desipramine, and atomoxetine demonstrated efficacy in improving ADHD symptoms in children with comorbid tics. In addition, alpha- 2 agonists and atomoxetine significantly improved comorbid tic symptoms. ${ }^{28}$ In the current systematic review, methylphenidate, clonidine, guanfacine, desipramine, and atomoxetine appear to reduce ADHD symptoms in children with tics, though the quality of the available evidence is low to very low. ${ }^{29}$ Therefore, GXR is not recommended for the treatment of ADHD in adults with comorbid tic disorder as first line therapeutic agent.

\section{Comparison Between ADHD Drugs}

Medications used to treat youths with ADHD have been studied extensively; however, direct comparisons between medications have not been investigated in head-to-head clinical trials. Faraone searched literature to identify double-blind, placebo-controlled trials in young people with ADHD from 1979 onwards that described the variability of drug-placebo effect sizes. ${ }^{30}$ Faraone conducted metaanalysis regression to assess the impact of medication type on pharmacological effects. The effect sizes for immediate-release (effect size 0.99) and long-acting stimulants (effect size 0.95 ) were broadly similar. On the other hand, the effect size for nonstimulants (effect size 0.57) was smaller than the effect sizes for stimulants. Similar investigations are needed for pharmacotherapy studies in adults with ADHD.

The abuse potential of stimulants is still controversially discussed. However, GXR has no abuse potential. This characteristic is one of the strengths of this medicine.

\section{Conclusion}

Guanfacine is an agonist that acts on $\alpha 2 \mathrm{~A}$-adrenoreceptors, that are highly concentrated in the locus coeruleus and prefrontal cortex. The beneficial actions arise from its ability to strengthen prefrontal cortical network connections to regulate attention, emotion, and behavior via its activity at postsynaptic $\alpha 2 \mathrm{~A}$ receptors. The current efficacy and safety evidence indicates that GXR as monotherapy is an effective treatment option for adults with ADHD.

\section{Abbreviations}

ADHD, attention-deficit/hyperactivity disorder; GXR, guanfacine extended-release; CYP, cytochrome p450; ADHD-RS, ADHD Rating Scale.

\section{Acknowledgments}

We thank Sarina Iwabuchi, PhD, from Edanz Group (https://en-author-services.edanz.com/ac) for editing a draft of this manuscript.

\section{Disclosure}

The authors declare that they have no conflicts of interest.

\section{References}

1. American Psychiatric Association. Diagnostic and Statistical Manual of Mental Disorders. 5th ed. Arlington: American Psychiatric Association; 2013.

2. Xu G, Strathearn L, Liu B, Yang B, Bao W. Twenty-year trends in diagnosed attention-deficit/hyperactivity disorder among US children and adolescents, 1997-2016. JAMA Netw Open. 2018;1(4):e181471. doi:10.1001/jamanetworkopen.2018.1471

3. Song P, Zha M, Yang Q, Zhang Y, Li X, Rudan I. The prevalence of adult attention-deficit hyperactivity disorder: a global systematic review and meta-analysis. $J$ Glob Health. 2021;11:04009. doi:10.7189/jogh.11.04009

4. Chai G, Governale L, McMahon AW, Trinidad JP, Staffa J, Murphy D. Trends of outpatient prescription drug utilization in US children, 2002-2010. Pediatrics. 2012;130(1):23-31. doi:10.1542/peds.2011-2879

5. Renoux C, Shin JY, Dell'Aniello S, Fergusson E, Suissa S. Prescribing trends of attention-deficit hyperactivity disorder (ADHD) medications in UK primary care, 1995-2015. Br J Clin Pharmacol. 2016;82 (3):858-868. doi:10.1111/bcp.13000 
6. Pliszka S; AACAP Work Group on Quality Issues. Practice parameter for the assessment and treatment of children and adolescents with attention-deficit/hyperactivity disorder. $J$ Am Acad Child Adolesc Psychiatry. 2007;46(7):894-921. doi:10.1097/chi.0b013e318 $054 \mathrm{e} 724$

7. Kooij SJ, Bejerot S, Blackwell A, et al. European consensus statement on diagnosis and treatment of adult ADHD: the European Network Adult ADHD. BMC Psychiatry. 2010;10:67.

8. Wolraich M, Brown L, Brown RT, et al.; Subcommittee on AttentionDeficit/Hyperactivity Disorder; Steering Committee on Quality Improvement and Management. ADHD: clinical practice guideline for the diagnosis, evaluation, and treatment of attention-deficit/hyperactivity disorder in children and adolescents. Pediatrics. 2011;128 (5):1007-1022

9. Bolea-Alamañac B, Nutt DJ, Adamou M, et al.; British Association for Psychopharmacology. Evidence-based guidelines for the pharmacological management of attention deficit hyperactivity disorder: update on recommendations from the British Association for Psychopharmacology. $J$ Psychopharmacol. 2014;28(3):179-203. doi:10.1177/0269881113519509.

10. Cortese S, Adamo N, Del Giovane C, et al. Comparative efficacy and tolerability of medications for attention-deficit hyperactivity disorder in children, adolescents, and adults: a systematic review and network meta-analysis. Lancet Psychiatry. 2018;5(9):727-738. doi:10.1016/ S2215-0366(18)30269-4

11. National Institute for Health and Care Excellence. Attention deficit hyperactivity disorder: diagnosis and management. NICE guideline [NG87]; 2018. Available from: https://www.nice.org.uk/ guidance/ng87/chapter/Recommendations. Accessed January 16, 2021 .

12. Bream JB, Lauener H, Picard CW, Scholtysik G, White TG. Substituted phenyl-acetyl guanidine: a new class of antihypertensive agents. Arzeimittel-forschung. 1975;25(10):1477-1482.

13. Doxey JC. Pre- and postsynaptic effects of alpha-agonists in the anococcygeus muscle of the pithed rat. Eur J Pharmacol. 1979;54 (1-2):185-189. doi:10.1016/0014-2999(79)90423-0

14. Westfall TC, Westfall DP. Adrenergic agonists and antagonists. In: Brunton LL, editor. Goodman \& Gilman's the Pharmacological Basis of Therapeutics. New York: McGraw-Hill; 2011:277-334.

15. Arnsten AF, Li BM. Neurobiology of executive functions: catecholamine influences on prefrontal cortical functions. Biol Psychiatry. 2005;57(11):1377-1384. doi:10.1016/j.biopsych.2004.08.019

16. Brozoski T, Brown RM, Rosvold HE, Goldman PS. Cognitive deficit caused by regional depletion of dopamine in prefrontal cortex of rhesus monkey. Science. 1979;205(4409):929-932. doi:10.1126/ science. 112679

17. Sagvolden T. The alpha-2A adrenoceptor agonist guanfacine improves sustained attention and reduces overactivity and impulsiveness in an animal model of attention-deficit/hyperactivity disorder (ADHD). Behav Brain Funct. 2006;2(1):41. doi:10.1186/1744-9081$2-41$
18. Easton N, Shah YB, Marshall FH, Fone KC, Marsden CA. Guanfacine produces differential effects in the frontal cortex compared with striatum: assessed by phMRI BOLD contrast. Psychopharmacology. 2006;189(3):369-385. doi:10.1007/s00213006-0558-1

19. Swartz BE, Kovalik K, Thomas K, Torgersen D, Mandelkern MA. The effects of an alpha2 adrenergic agonist, guanfacine, on rCBF in human cortex in normal controls and subjects with focal epilepsy. Neuropsychopharmacology. 2000;23(3):263-275. doi:10.1016/ S0893-133X(00)00101-9

20. Cruz MP. Guanfacine extended-release tablets (Intuniv), a nonstimulant selective alpha2a-adrenergic receptor agonist for attention-deficit/hyperactivity disorder. $P$ T. 2010;35(8):448-451.

21. Bukstein OG, Head J. Guanfacine ER for the treatment of adolescent attention-deficit/hyperactivity disorder. Expert Opin Pharmacother. 2012;13(15):2207-2213. doi:10.1517/14656566.2012.721778

22. Stahl SM. Stahl's Essential Psychopharmacology: Neuroscientific Basis and Practical Applications. New York: Cambridge University Press; 2008.

23. Butterfield ME, Saal J, Young B, Young JL. Supplementary guanfacine hydrochloride as a treatment of attention deficit hyperactivity disorder in adults: a double blind, placebo-controlled study. Psychiatry Res. 2016;236:136-141. doi:10.1016/j.psychres.2015. 12.017

24. Iwanami A, Saito K, Fujiwara M, Okutsu D, Ichikawa H. Efficacy and safety of guanfacine extended-release in the treatment of attention-deficit/hyperactivity disorder in adults: results of a randomized, double-blind, placebo-controlled Study. J Clin Psychiatry. 2020a;81(3):19m12979. doi:10.4088/JCP.19m12979

25. Iwanami A, Saito K, Fujiwara M, Okutsu D, Ichikawa H. Safety and efficacy of guanfacine extended-release in adults with attention-deficit/hyperactivity disorder: an open-label, long-term, phase 3 extension study. BMC Psychiatry. 2020b;20(1):485. doi:10.1186/s12888-020-02867-8

26. Shire Canada Inc. Intuniv XR product monograph. Ville SaintLaurent, QC: Shire Canada Inc. 2013.

27. Scahill L, McCracken JT, King BH, et al. Extended-release guanfacine for hyperactivity in children with autism spectrum disorder. Am J Psychiatry. 2015;172(12):1197-1206. doi:10.1176/appi.ajp.2015.15010055

28. Bloch MH, Panza KE, Landeros-Weisenberger A, Leckman JF. Metaanalysis: treatment of attention-deficit/hyperactivity disorder in children with comorbid tic disorders. $J$ Am Acad Child Adolesc Psychiatry. 2009;48(9):884-893.

29. Osland ST, Steeves TDL, Pringsheim T. Pharmacological treatment for attention deficit hyperactivity disorder (ADHD) in children with comorbid tic disorders. Cochrane Database Syst Rev. 2018;2018(6): CD007990.

30. Faraone SV. Using meta-analysis to compare the efficacy of medications for attention-deficit/hyperactivity disorder in youths. $P T$. 2009;34(12):678-694.

\section{Publish your work in this journal}

Drug Design, Development and Therapy is an international, peerreviewed open-access journal that spans the spectrum of drug design and development through to clinical applications. Clinical outcomes, patient safety, and programs for the development and effective, safe, and sustained use of medicines are a feature of the journal, which has also been accepted for indexing on PubMed Central. The manuscript management system is completely online and includes a very quick and fair peer-review system, which is all easy to use. Visit http://www. dovepress.com/testimonials.php to read real quotes from published authors. 